\title{
Correction: Retinopathy of prematurity in Rwanda: a prospective multi-centre study following introduction of screening and treatment services
}

\author{
Francis Mutangana ${ }^{1} \cdot$ Charles Muhizi $^{2} \cdot$ Godelieve Mudereva $^{3} \cdot$ Piet Noë $^{4} \cdot$ Stephenson Musiime $^{5}$. \\ Tharcisse Ngambe $^{5} \cdot$ Lisine Tuyisenge ${ }^{6} \cdot$ Muzungu Kumwami $^{6} \cdot$ Hovaire Nsabimana $^{7} \cdot$ Craig Conard $^{7}$. \\ Yannick Fonteyne ${ }^{8} \cdot$ Ingele Casteels $^{9} \cdot$ Stefan De Smedt $^{10} \cdot$ for the Rwanda Retinopathy of Prematurity Study Group
}

Published online: 4 September 2019

(c) The Author(s), under exclusive licence to The Royal College of Ophthalmologists 2019

\section{Correction to: Eye \\ https://doi.org/10.1038/s41433-019-0529-5 \\ Published online 30 July 2019}

In the original Article, Erwin Van Kerschaver was erroneously attributed an affiliation. The publishers would like to apologies for this error. This has been corrected in the XML, HTML and PDF versions of this Article. The Publishers would like to apologise for the error.

Acknowledgements The authors would like to acknowledge the members of the Rwanda Retinopathy of Prematurity (ROP) Study Group for their valuable contribution to this study (alphabetical order): Cannoodt Luk, PhD, Health Policy Analyst, KU Leuven (Belgium), NGO Umubano-Impore; Casteels Ingele, $\mathrm{MD}, \mathrm{PhD}$, Paediatric Ophthalmologist, KU Leuven, Belgium; Conard Craig, MD, MPH, Paediatrician CHUB; De Smedt Stefan, MD, PhD, ophthalmologist/epidemiologist, AZ St Maarten, Mechelen, Belgium; Devlieger Hugo, MD, PhD, neonatologist, KU Leuven, Belgium; Fonteyne Yannick, MD; Gilbert Clare, MD, PhD, International Centre for Eye Health, London School of Hygiene and Tropical Medicine, London, UK; Gisagara Egide, MD, ophthalmologist ; Kumwami Muzungu, MD, paediatrician CHUK; Mudereva Godelieve, MD, ophthalmologist CHUK; Muhizi Charles, MD,

These authors contributed equally: Francis Mutangana, Charles Muhizi

\footnotetext{
Stefan De Smedt, MD PhD

dr.stefan.desmedt@gmail.com

1 Ophthalmology, King Faisal Hospital, Kigali, Rwanda

2 Ophthalmology, CHUB, Huye, Rwanda

3 Ophthalmology, CHUK, Kigali, Rwanda

4 Ophthalmology, Kabgayi Eye Hospital, Rwanda Charity Eye Hospital, Southern Province, Rwanda
}

ophthalmologist CHUB; Mulindwa Karemazi Gustave, MD, paediatrician CHUB; Musiime Stephenson, MD, FCPaeds(SA), paediatrician KFH; Mutangana Francis, MD, ophthalmologist KFH; Mutsinzi Edison, MD, ophthalmologist Kabgayi Eye Unit; Ngambe Tharcisse, MD, paediatrician KFH; Noë Piet, MD, ophthalmologist Kabgayi Eye Unit, Rwanda Charity Eye Hospital; Nsabimana Hovaire, MD, paediatrician CHUB; Nyemazi Alex, MD, ophthalmologist KFH; Sebuseruka Sonia, MD, ophthalmologist; Tuyisabe Theophile, MD, ophthalmologist Kabgayi Eye Unit; Tuyisenge Lisine, MD, paediatrician CHUK; Uwurukundo Marie Claude, MD, paediatrician KFH; Van Kerschaver Erwin, MD PhD.

Rwanda Retinopathy of Prematurity (ROP) Study Group Luk Cannoodt $^{11}$, Ingele Casteels ${ }^{9}$, Craig Conard ${ }^{7}$, Stefan De Smedt ${ }^{10}$, Hugo Devlieger ${ }^{12}$, Yannick Fonteyne ${ }^{8}$, Clare Gilbert ${ }^{13}$, Egide Gisagara $^{3}$, Muzungu Kumwami ${ }^{6}$, Godelieve Mudereva ${ }^{3}$, Charles Muhizi $^{2}$, Gustave Mulindwa Karemazi ${ }^{7}$ Stephenson Musiime ${ }^{5}$, Francis Mutangana ${ }^{1}$, Edison Mutsinzi ${ }^{14}$, Tharcisse Ngambe, Piet Noë ${ }^{15}$, Hovaire Nsabimana ${ }^{7}$, Alex Nyemazi ${ }^{1}$, Sonia Sebuseruka ${ }^{16}$, Theophile Tuyisabe $^{14}$, Lisine Tuyisenge ${ }^{6}$, Marie Claude Uwurukundo ${ }^{5}$, Erwin Van Kerschaver

\footnotetext{
${ }^{11}$ Health Policy Analist, NGO Umubano-Impore, KU Leuven, Leuven, Belgium; ${ }^{12}$ Pediatrics, KU Leuven, Leuven, Belgium; ${ }^{13}$ International Centre for Eye Health, Department of Clinical Research, London School of Hygiene and Tropical Medicine, London, UK; ${ }^{14}$ Ophthalmology, Kabgayi Eye Unit, Muhanga, Rwanda; ${ }^{15}$ Ophthalmology, Kabgayi Eye Unit, Rwanda Charity Eye Hospital, Southern Province, Rwanda; ${ }^{16}$ Ophthalmology, Kigali, Rwanda
}

5 Pediatrics, King Faisal Hospital, Kigali, Rwanda

6 Pediatrics, CHUK, Kigali, Rwanda

7 Pediatrics, CHUB, Huye, Rwanda

8 External consultant, Mechelen, Belgium

9 Ophthalmology, KU Leuven, Leuven, Belgium

10 Ophthalmology, Epidemiology, AZ St Maarten Hospital, Mechelen, Belgium 